\title{
Les métamorphoses de la critique littéraire : le cas des Grands Rhétoriqueurs
}

\author{
The metamorphoses of literary criticism: the case \\ of the Grands Rhétoriqueurs
}

Dorota Szeliga

Université de Varsovie, Pologne

\begin{abstract}
Résumé : L'analyse de l'attitude des critiques à l'égard de la poésie des Grands Rhétoriqueurs permet d'observer les changements intervenus au sein de l'histoire littéraire. Longtemps méprisés et pratiquement exclus de la liste des poètes importants, les Rhétoriqueurs ont enfin su gagner la bienveillance des chercheurs et retrouver, dans la seconde moitié du XXe siècle, leur place dans I'histoire de la littérature française. Ce changement notoire stimulé de nouvelles recherches dans des champs de la littérature complètement oubliés jusqu'alors.

Mots-clés : poésie, critique littéraire, renaissance, rhétoriqueurs, Puy.
\end{abstract}

Abstract: The analysis of the attitude of the critics towards the poetry of the Grands Rhétoriqueurs allows us to observe the changes that have occurred within literary history. Long despised and practically excluded from the framework of important poets, the Rhétoriqueurs finally won over the benevolence of researchers and in the second half of the 20th century, they found their place in the history of French literature. It was a noticeable change that spurred new research into previously forgotten fields of literature.

Keywords: poetry, literary criticism, renaissance, rhetoric, Puy.

Les Grands Rhétoriqueurs, ces «mal aimés » de la critique comme les a appelés Paul Zumthor ${ }^{1}$, jouissent enfin d'un moment favorable pour leur œuvre. Néanmoins, pour que ce changement d'attitude ait été possible, il a fallu que la critique littéraire passe par des transformations profondes. Le chemin fut long et difficile. L'évolution de la critique envers les poètes dont l'activité s'étend des quarante dernières années du XVe siècle aux trente premières du XVle, appelés Grands Rhétoriqueurs d'après la tradition littéraire, fut lente et il faut attendre les années 1960 du XXe siècle pour voir apparaître des tendances favorables à leur égard.

Leur infortune a commencé au moment du triomphe de la Pléiade, qui lançait un nouveau programme poétique dont l'esthétique de la grande rhétorique fut définitivement exclue. Dans la Défense et illustration de la langue française,

\footnotetext{
1 C'est ainsi que Paul Zumthor commence son étude : «Les mal aimés. Les mal nommés, d'un terme confectionné au XIXe siècle par quelque historien inattentif, puis consacré par les lexicographes » (Zumthor, 1978b : 9).
} 
manifeste du groupe, Joachim Du Bellay se déchaîne contre les rhétoriqueurs, contrairement à Thomas Sébillet, auteur d'un Art poétique français, qui se montre plutôt favorable à leur poésie. L'édition du traité de Sébillet a précédé de quelques mois celle du manifeste du groupe de jeunes poètes organisé par Pierre de Ronsard. Deux textes, deux optiques différentes, deux conceptions opposées du développement de la poésie. Sébillet opte pour une évolution harmonieuse de la poésie française, tandis que Du Bellay propose une rupture. Sébillet cite volontiers les poètes français d'autrefois dans le but «-de donner des modèles de ce que les Français ont déjà su faire, avec leurs propres moyens, en matière d'harmonie et de varietas " (Goyet, 1990 : 17). Les noms d'Alain Chartier, de Mellin de Saint-Gelais et de Jean Lemaire de Belges reviennent presque aussi souvent que ceux de Clément Marot et de Maurice Scève. Sébillet s'insurge contre l'usage consistant à appeler les anciens auteurs français "rimeurs ", car selon lui, ils méritent bien le nom de poètes. Tout en appréciant le sonnet et l'ode, il ne délaisse pas pour autant les genres tels que la ballade, le chant royal ou le rondeau, que Du Bellay traitera d'u-episseries "), parlant avec mépris de toute la poésie de ses devanciers dans la Défense. En effet, depuis la génération de la Pléiade, les rhétoriqueurs ont mauvaise presse. Très vite oubliés, ils ne suscitent guère d'intérêt jusqu'à la deuxième moitié du XIXe siècle lorsqu'est forgée l'appellation de rhétoriqueurs, à connotation péjorative, qui a marqué pour longtemps leur destin. Cette expression mal fondée se trouve déjà dans l'Histoire de la Langue et de la Littérature française des origines à 1900 publié sous la direction de Petit de Julleville. Édouard Bourciez, professeur à la Faculté des lettres de l'Université de Bordeaux, chargé de rédiger le passage concernant la problématique de la poésie du début du XVIe siècle, n'a pas beaucoup d'indulgence pour cette période de la littérature française :

Sous l'influence d'Alain Chartier, il s'était formé une école savante qui fleurit d'abord à la cour de Bourgogne et, un peu plus tard, dans les Flandres, gouvernées par Marguerite d'Autriche. Les représentants de cette école eurent presque tous la prétention d'être à la fois orateurs, historiens et poètes: mais leur éloquence ne fut qu'une emphase ridiculement boursouflée, leur poésie rampa au milieu d'allégories morales froidement délayées ; d'historiens, ou même d'historiographes, ils n'eurent que le nom, et furent des apologistes aux gages des princes qui les rémunéraient. Leur groupe est connu sous le nom qu'ils s'étaient décerné eux-mêmes comme un titre d'honneur, et qui caractérise bien leur manière: ce sont « les grands Rhétoriqueurs $॥$ (Bourciez, $1897: 85$ ).

En 1910, Henri Guy, dans son Histoire de la poésie française au XVle siècle, a employé le terme abusif d'"école des Rhétoriqueurs» qui s'est implanté dans les milieux de la critique, ce dont les conséquences se font sentir encore aujourd'hui. Ce terme impropre est à l'origine de plusieurs malentendus. En fait, il s'agit d'au moins deux générations de poètes qui n'avaient pas conscience d'appartenir à un groupe organisé, encore moins à une école. Ils n'ont laissé aucun manifeste ni témoignage d'un programme, et n'ont pas eu de maître qu'ils auraient voulu suivre ou imiter de manière méthodique. Les relations personnelles ou épistolaires que quelques-uns ont entretenues ne dépassent pas le cadre d'échanges intellectuels, ce qui ne suffit pas pour en déduire l'existence d'un groupe formé autour d'un programme établi. Le nombre des poètes regroupés sous cette appellation varie d'ailleurs selon les critiques, allant de douze à quelque quarante noms. 
Quant au terme de rhétoriqueurs, Paul Zumthor a déjà précisé que les poètes eux-mêmes ne l'avaient employé que très rarement, lui préférant les noms de "rhétoriciens ", de "rimeurs" et d'" orateurs" (Zumthor, 1978b:9). Notons que la première attestation du syntagme "grans rethoriqueurs" se trouve sans doute dans un passage des Droitz nouveaulx de Guillaume Coquillart, dans une série qui désigne, de manière plaisante, "les maîtres rusés de la parole " (Cerquiglini, $1997: 75-82)^{2}$. Cette plaisanterie, devenue étiquette à la suite des travaux de Charles d'Héricault3, s'est vite transformée en appellation péjorative, ce qui n'est pas étonnant vu l'incompréhension et le mépris qui régnaient dans ces publications :

\begin{abstract}
On devine qu'au milieu de ces complications de la forme, de ces procédés enfantins - allitérations, répétitions ineptes de syllabes, batteries de mots plus ou moins sonores, - il n'est nulle part question du fond. Voilà où l'on en était encore pendant les premières années du règne de François ler: le moyen âge se survivait au milieu de ces raffinements du mauvais goût, et la pensée risquait à son tour de se stériliser, grâce à cette ridicule technique, à tous ces préceptes que Marot lui-même a parfois trop docilement suivis (Petit de Julleville, $1897: 87-88$ ).
\end{abstract}

Henri Guy ne fait que suivre cette voie de la critique qui, faute d'instruments permettant d'aborder le texte d'un rhétoriqueur, s'en détourne avec dégoût. Cependant, on constate ici une différence: si Bourciez n'a consacré aux Grands Rhétoriqueurs qu'une dizaine de pages, Guy leur a réservé un volume entier. Ses recherches dans le domaine de la grande rhétorique ont constitué pour un demisiècle environ un modèle d'approche obligatoire, d'autant plus qu'au niveau des données factuelles ses travaux restent souvent exacts. Comment ne pas être déconcerté devant cet aveu du critique, qui, après avoir étudié avec tant de soin les œuvres d'une quarantaine d'auteurs, constate, résigné, dans sa conclusion :

II me semble que rien ne serait plus inutile ni plus fastidieux que de montrer ici, d'une façon résumée, en quoi la poésie des rhétoriqueurs est mauvaise. J'espère que les pages (tant de pages !) que je leur ai consacrées ont mis ce point en lumière, et je veux maintenant dire en quelques mots pourquoi leur art était condamné à rester difforme et stérile (Guy, 1910 : 377).

Guy leur reproche "un imperturbable dogmatisme ", "la paresse de l'esprit ", un optimisme béat, l'indifférence à l'injustice sociale, l'incompréhension des transformations du monde qui s'opèrent devant leurs yeux et auxquelles ils sont insensibles, la servilité envers les seigneurs qui étaient leurs mécènes. Cependant, ce qui, aux yeux du critique, était surtout impardonnable, c'est le rôle qu'ils avaient assigné à la poésie: une fonction descriptive de la réalité, subordonnée aux exigences du moment, voire à la politique des princes. Leur poésie ne serait donc

\footnotetext{
2 Voici le passage concerné :

Venez, venez sophistiqueurs,

Gens instruits, plaisans topiqueurs,

Rempliz de cautelles latentes,

Expers, habilles decliqueurs

Orateurs, grans rethoriqueurs

Garnis de langues esclatantes.

Droitz nouveaulx (vv. 13-18)
}

3 Ch. d'Héricault a employé ce terme dans son introduction au recueil d'E. Crépet (1861) : Les poètes français: recueil des chefs-d'œuvre de la poésie française depuis les origines jusqu'à nos jours, avec une notice littéraire sur chaque poète, t. I: Du XIIe au XVIe siècle. Paris. 
formée que de pièces de circonstance sans prétention à interpréter le monde dans lequel ils vivaient ou, pis encore, de pièces historiques déformant l'histoire en vue de plaire au prince. La forme des œuvres ne serait ainsi que la conséquence de cette mission attribuée de manière erronnée au poète :

Les poètes ont les rythmes et les allégories qu'ils méritent. Ceux-ci, pauvres de sentiment et plus encore d'idées, ne s'intéressent, comme Brind'oison, qu'à la forme, et c'est justement parce qu'ils n'ont rien à dire, qu'ils parlent avec tant de recherche. Faute de pensées à exprimer, ils s'amusent à combiner des sons, et croient qu'on ne s'apercevra point, s'ils jettent cette poudre aux yeux, de l'indigence de leurs conceptions. Mais le remède est pire que le mal, et ils tombent de Charybde en Scylla (Guy, $1910: 381$ ).

Les études de Guy ont canalisé pour longtemps les recherches sur les Grands Rhétoriqueurs, produisant une impression qui décourageait les chercheurs plutôt qu'elle ne les inspirait. Ainsi, une constatation amère de François Rigolot paraît tout à fait juste: « Rares ont été, avant les années 1970, ceux qui ont osé trouver le moindre intérêt à ces parias de l'écriture»" (Rigolot, 1993 : 124). Ce n'est pas uniquement le manque d'intérêt pour cette période de la poésie : cela tient aussi à une certaine infamie qui a frappé ce champ de recherche. D'une part, le problème semblait avoir été examiné de manière exhaustive par Henri Guy; d'autre part, une interprétation différente de la sienne paraissait impossible et aurait pu compromettre le goût esthétique d'un critique qui aurait regardé d'un œil bienveillant les œuvres des Rhétoriqueurs.

Il faut cependant noter que Guy a fait plusieurs observations qui restent valables malgré leur caractère tendencieux. Ne disposant pas d'appareil critique approprié, il reste désarmé devant ces textes difficiles dont l'esthétique et la portée étaient trop éloignées de la sensibilité et des canons artistiques en vigueur au début du XXe siècle. Tout en présentant minutieusement les auteurs, leurs vies et leurs œuvres, leurs procédés littéraires, il se perd dans des interprétations qui pèchent par leur manque d'objectivité. On lui doit la consécration du terme d'uécole des Rhétoriqueurs " ainsi qu'une distinction vague entre les grands et petits rhétoriqueurs ${ }^{4}$. II reconnaît l'existence d'une certaine poétique caractéristique des œuvres du groupe, mais il y voit justement la source de leur échec. Si certains des petits rhétoriqueurs ont réussi, c'est

Parce qu'ils ont eu la chance de travailler à l'écart, de s'affranchir - au moins en partie - des règles et des modes qui abêtissaient les prétendus habiles, les écrivains patentés (Guy, $1910: 317-318$ ).

Aux yeux du critique, les jeux de langage pratiqués par ces poètes témoignent uniquement de leur focalisation sur l'aspect formel de la poésie, voire sur sa fonction secondaire et complémentaire par rapport au message. L'habileté de l'expression ne doit jamais étouffer la richesse du contenu. Si la forme l'emporte sur le contenu, celui-ci devait être de peu de poids.

\footnotetext{
4 «J'appelle 'petits rhétoriqueurs' et ceux qui n'ont laissé que peu d'œuvres, et ceux dont les contemporains n'ont pas fait, ce semble, un très grand cas. Mais comme le mérite d'un poète ne dépend en rien du nombre de ses vers, et que, d'autre part, le public était hors d'état, au commencement du XVIe siècle, de discerner le meilleur du pire, il s'ensuit que les petits rhétoriqueurs ne sont pas nécessairement les moindres ॥ (Guy, $1910: 317-318$ ).
} 
À ces accusations un peu hâtives de manque de lyrisme, s'ajoutent les reproches de caractère idéologique. Guy s'indigne de la prétendue indifférence des Rhétoriqueurs aux malheurs du menu peuple, voire aux inégalités sociales. Toutefois, à la lumière des recherches récentes, cette optique se révèle anachroniques: le critique, fasciné par l'idée de progrès, notion magique au début du XXe siècle, ne veut pas reconnaître aux poètes le droit d'avoir une autre vision du monde, dans laquelle cette notion n'avait pas sa place. Selon Guy, l'observation et la description du mal social devaient logiquement mener à la décision de transformer la société, quel que soit le prix du bouleversement de l'ordre social. Pour les Rhétoriqueurs en revanche, ce qui comptait c'était l'harmonie dans la vie sociale. S'ils envisagent des changements, c'est surtout dans le cadre du perfectionnement du système dans lequel ils vivent. L'excuse que Guy trouve pour expliquer la raison de ce prétendu non-engagement est leur dépendance financière vis-à-vis des princes: «Pas de lovanges, pas de pensions, telle est la loi d'airain de ce temps-là, et les écrivains qui l'ont subie paraissent plus à plaindre qu'à blâmer " (Guy, 1910:378-379). Ce jugement, aussi injuste soit-il, a longtemps perduré. Une soixantaine d'années plus tard, Paul Zumthor l'a repris pour en tirer d'autres conséquences, mais en partant du même point de départ, à savoir le statut social des poètes.

Par ailleurs, Guy ne dissimule pas les motifs qui l'ont poussé à travailler sur ce champ ingrat de la littérature qu'était alors l'œuvre des Rhétoriqueurs. Tout d'abord, il voulait mettre l'accent sur le changement qui s'était opéré dans la critique littéraire :

Si j'ai écrit ce volume, c'est que le temps n'est plus, il me semble, où la critique littéraire, servante de la pure esthétique, ne s'occupait que des choses belles, et ne recherchait, parmi les ouvrages d'autrefois, que des sujets d'admiration. Aujourd'hui, c'est l'histoire de la pensée humaine que nous désirons connaître, et, en ce sens, les déviations ou les défaillances de cette pensée nous intéressent presque autant que sa marche normale ou ses coups d'aile (Guy, $1910: 382$ ).

Il a pressenti l'importance de leur poésie plutôt qu'il ne l'a reconnue, mais il a fait un effort d'objectivité en reconnaissant le rôle des Rhétoriqueurs dans la promotion de la littérature antiqueb. Ainsi, ces "premiers ouvriers de la Renaissance" préparaient l'arrivée de la Pléiade. Leurs œuvres, aussi imparfaites qu'elles soient,

\footnotetext{
5 Voir par exemple l'opinion de J. Devaux, qui analyse l'attitude de Jean Molinet par rapport à la guerre et prouve qu'il est loin de défendre uniquement la cause de celui qui lui verse des gages. Chroniqueur et poète, ce dernier se sert de rhétorique pour attirer l'attention des grands sur le sort des povres gens. J. Devaux conclue: «Si l'indiciaire se faisait là l'interprète des aspirations profondes de la population, ce discours n'allait pas, néanmoins, sans quelque audace. J. Molinet, rappelons-le, compose le Temple de Mars en un temps où son prince, Charles le Téméraire, ne cesse de guerroyer, en Allemagne ou en Lorraine. Plus encore, dans la Ressource, il ose prendre à partie les princes puissans, roix et ducs, en l'occurence son propre maître, l'archiduc Maximilien, alors en guerre contre le roi Louis de France "); Devaux, J. (1997: 116) : Rhétorique et pacifisme chez Jean Molinet. in Grands Rhétoriqueurs.

6 «En fait, ces ridicules versificateurs qui vécurent au temps de Charles VIII et de Louis XII ont été les premiers ouvriers de la Renaissance. Eł savez-vous comment ils la préparaient ? En aimant l'antiquité avec passion. A leurs yeux chaque livre grec ou latin fut une sorte de Bible. lls vénéraient de confiance, commentaient, traduisaient vaille que vaille ces textes essentiels, monuments de mille années de sagesse, et leur vouaient un culte d'autant plus fanatique qu'ils ne pouvaient les entendre pleinement. Touchante, respectable idolâtrie. Si ces pauvres d'esprit ne l'avaient pas eve, jamais n'auraient brillé les sept étoiles de la Pléiade » (Guy, $1910: 381$ ).
} 
auraient servi de point de référence à celles d'un Ronsard ou d'un Du Bellay, en dépit de leur infériorité littéraire incontestable. Guy a également souligné l'importance de leur activité pour la vie intellectuelle et spirituelle de l'époque.

La fin des années cinquante et les années soixante ont vu un certain regain d'intérêt pour la poésie des Rhétoriqueurs, grâce à Albert-Marie Schmidt?7. Son étude a frayé le chemin à d'autres recherches, éditions ou études thématiques qui, ayant définitivement rompu avec les interprétations du début du siècle, ont essayé de réhabiliter ce groupe d'écrivains. Le chapitre "L'Age des Rhétoriqueurs », rédigé par A.-M. Schmidt pour la Bibliothèque de la Pléiade (1958), a ouvert la voie à une analyse bienveillante et nuancée des œuvres des Rhétoriqueurs. Le critique y souligne l'importance de leur apport original à la littérature française, de la vulgarisation des idées humanistes aux recherches formelles dans la poésie. Leur engagement dans les affaires publiques est interprété comme le signe d'une sensibilité nouvelle du citoyen, attitude qu'ils cherchent à propager :

\begin{abstract}
Préoccupés d'acheminer l'histoire vers un heureux terme, les humanistes rhétoriqueurs, loin de chercher à s'abstraire du cours de choses, s'y engagent de leur mieux. Ils ne se réfugient pas dans un jardin clos ou dans une tour d'ivoire. S'ils ne parviennent pas à expliquer tous les événements, du moins s'appliquent-ils à les définir, à les contrôler, à les enregistrer, à les réduire en mythes, à leur attribuer un sens éthique dans le fallacieux espoir qu'ils l'assumeront (Schmidt, 1958 : 176-177).
\end{abstract}

Schmidt apprécie également la prudence des Rhétoriqueurs dans le renouvellement de la poésie. Ils procèdent à des changements tout en respectant les principes médiévaux, ce qui ne les empêche pas de devenir des "expérimentateurs du langage ". La décision de se tourner vers la sonorité des poèmes s'est avérée fructueuse. Les jeux polyphoniques, de rimes et de rythmes, contribuent à rendre le message poétique plus expressif. Cette poésie, qui privilégie la réflexion sur les questions sociales, religieuses et scientifiques, n'échappe pas à la tentation didactique. Cependant, dans leurs recherches, les poètes vont jusqu'à un certain surréalisme (Schmidt, 1958 : 187).

Dans ce contexte apparaissent des éditions nouvelles des textes des Rhétoriqueurs, parmi lesquelles La Concorde du Genre humain de Jean Lemaire de Belges éditée par P. Jodogne (1964), suivie d'une étude: Jean Lemaire de Belges, écrivain franco-bourguignon (1972); OEuvres de Jean Robertet par M. Zsuppan (1970) ; Les Lunettes des Princes de Jean Meschinot par Ch. Martineau-Genieys (1972) ; Le Voyage de Gênes de Jean Maroł par G. Trisolini (1974)8. Parmi les études thématiques les plus intéressantes, il faut citer celle de Françoise Joukovsky : La gloire dans la poésie française et néolatine du XVle s. (des Rhétoriqueurs à Agrippa d'Aubigné) (1969), et le travail de Ch. Martineau-Genieys : Le thème de la mort dans la poésie française de 1450 à 1550.

Cependant, c'est l'ouvrage de Paul Zumthor: Le masque et la lumière, qui a le plus attiré l'attention des critiques, ainsi que celle d'un public plus large. Constituant

\footnotetext{
7 Voir « L'Age des Rhétoriqueurs », dans le chapitre «La Littérature humaniste à l'époque de la Pléiade », Histoire des littératures (1958 : 175-190), t. III, Gallimard, Bibliothèque de la Pléiade.

8 Voir également une étude récente de Jean-Luc Nardone, « II ritratto di Genova negli scritti del 1507 di Jean Marot, storico della regina di Francia, e di Giacomo de' Sorci detto il Cortonese ", Cahiers des recherche médiévales et humanistes, 2019/2, 38, pp. 175-200.
} 
sans-doute une sorte de provocation, ce livre est devenu, depuis sa parution en 1978, une référence pour ceux qui s'intéressent à la grande rhétorique. En effet, l'application de la linguistique saussurienne et des théories structuralistes à une analyse des œuvres des Rhétoriqueurs a généré plusieurs découvertes, et grâce à cette approche nouvelle, la critique a eu enfin à sa disposition une clé facilitant la compréhension de leurs textes. Dans cette perspective, l'aspect formel des poèmes a cessé d'être un inconvénient: au contraire, il est devenu un élément privilégié d'analyse pour la critique des années soixante-dix. Si les propositions de Zumthor invitant à s'ouvrir à la langue et au style des Rhétoriqueurs ont été accueillies avec enthousiasme, sa thèse concernant les origines de la fascination des poètes pour la richesse de la forme: de l'allégorie, en passant par l'ornement, jusqu'à la rime, a soulevé, dès le début, plusieurs polémiques. Selon Zumthor :

\begin{abstract}
La véritable question qu'au lecteur d'aujourd'hui posent ces textes s'articule donc au niveau de leur production. De ce point de vue, les rhétoriqueurs apparaissent, collectivement, comme obsédés par la recherche d'un mode d'écrire qui permette de désaliéner et de repersonnaliser le rapport de l'écrivain à son écriture. Au sein d'un monde princier qui faisait profession d'immutabilité et où toute existence spontanément tournait en spectacle, les rhétoriqueurs tentèrent de faire, du langage même, dans la materialité de ses structures propres (sonores, lexicales, rythmiques), le seul spectacle vrai et le seul acteur (Zumthor, 1978a: 12-13).
\end{abstract}

Dans cette conception, le rapport de subordination contraignant du poète vis-àvis du prince reste un élément essentiel et participe d'un essai de réinterprétation de l'opinion de Guy, qui considère la production des Rhétoriqueurs comme une résultante de la dépendance financière du poète de cour. Cependant, alors que ce dernier voit en cette dépendance la source de la médiocrité de la poésie des Rhétoriqueurs, Zumthor identifie en elle ce qui motive leurs expériences linguistiques. En dépit de la perspicacité de cette analyse, il faut reconnaître qu'elle pèche par un certain anachronisme et des inexactitudes signalés d'ailleurs par Claude Thiry dans ses textes polémiques ${ }^{9}$. Comme toute thèse séduisante, celle de Zumthor met surtout en avant, parmi les données des recherches récentes, celles susceptibles de donner au texte rhétoriqueur "l'apparence de l'actualité " (Grands Rhétoriqueurs, 1997:7). D'une part, l'auteur s'adresse à un public déjà familiarisé avec les expériences linguistiques du surréalisme ou du mouvement Dada, d'autre part il exploite les méthodes de l'analyse textuelle fondée sur les théories de Freud ou Lacan. L'aliénation du poète due au malaise produit par le fait de vivre dans une société se nourrissant des inégalités constitue toutefois une vision trop simplificatrice des relations sociales caractéristiques de la société à la fin du XVe et au début du $X V \mid$ le siècle. En fait, le prétendu principe de relation entre le prince lou un autre "grand» protecteur) et le poète, ce principe de servilité découlant de l'état de dépendance économique aurait rendu impossible tout mécénat. Si chaque aide financière avait impliqué la servitude intellectuelle, les artistes n'auraient pas créé tant d'œuvres qui continuent de fasciner jusqu'à nos jours. Nous savons que la relation mécène-artiste est beaucoup plus nuancée. Cette proposition de voir dans

\footnotetext{
9 Voir C. Thiry (1980). La poétique des Grands Rhétoriqueurs. À propos d'un ouvrage récent . Le Moyen Age, 86 (1), 117-133; et id. (1978). Lecture du texte de "rhétoriqueur », Cahiers d'Analyse textuelle, 20, 85-101.
} 
le couple prince-poète un reflet des tensions sociales clarifie sans doute l'exposé, mais fait obstacle à une bonne interprétation de plusieurs textes et à l'évaluation de leur portée. Michel Zink a ainsi résumé l'importance des recherches de Zumthor :

Tout en étant très attentif au statut social des Rhétoriqueurs, à leur situation professionnelle et même financière dans les cours princières du XVe siècle, Zumthor soulignait surtout ce vertige du langage qui, de façon en apparence structuraliste, jove sur le rapport phonique ou métrique des mots entre eux plus que sur leur sens, s'enferme dans l'infini limité des échos ou des miroirs parallèles, se fonde sur ce qu'on appelait à l'époque «les jeux du signifiant ॥ (Grands Rhétoriqueurs, 1997 : 7).

Certes, en ces années 1970 du XXe siècle, le terme d'aliénation et l'évocation du non-conformisme dissimulé sous le masque du "discours de la Gloire " devait répondre à "l'horizon d'attente " du public. Dans son étude, Zumthor ne cache pas d'ailleurs qu'il veut "re-situer [le texte] dans une lumière qui le rende compréhensible pour nous " (Zumthor, 1978a: 8). "l élabore même le type du poète rhétoriqueur, lequel ressemble assez à un intellectuel pratiquant l'émigration intérieure - rapprochement intéressant à cela près qu'il n'est pas applicable à un grand nombre de textes.

Thiry, qui choisit une approche différente, reconnaît que le mérite de Zumthor consiste à «-avancer une explication de l'écriture de ces auteurs si vivement intéressés par l'exploration du langage » (Thiry,1980:86), mais il propose une autre voie d'interprétation du phénomène « rhétoriqueur $»$ :

\begin{abstract}
Restreindre le champ d'action des rhétoriqueurs à la seule littérarité - et surtout, au sein de celle-ci, à un travail sur le langage conçu comme fin en soi et échapatoire à une condition intellectuelle [...] c'est en effet faire trop bon marché de tout ce qui, dans la production sérieuse du groupe, réfère à I'histoire, à l'actualité, à la politique, tout ce qui est, si l'on veut, " thématique ", c'est-à-dire en fait tout ce qui constitue la mission première des rhétoriqueurs (Thiry, $1980: 130$ ).
\end{abstract}

Selon Thiry, c'est à Paul Jodogne qu'on doit la meilleure définition de cette mission première des Rhétoriqueurs:

Ces écrivains se considèrent en quelque sorte comme responsables de l'ordre religieux, politique, moral et culturel non seulement de leur cercle social, mais de leur nation, voire de l'ensemble de la Chrétienté. En vérité, leur conscience d'écrivains est profondément ambitieuse (Jodogne, $1970: 161)$.

Cette approche a ouvert de nouveaux horizons dans la recherche sur les Rhétoriqueurs. Thiry et d'autres critiques comme Christine Martineau-Génieys ont essayé de replacer leur statut dans une perspective historique, en évitant deux clichés : le rapport de subordination qui ne laisse aucune liberté aux écrivains gagés, et le "désengagement» nécessaire et inévitable du poète de cour vis-à-vis de sa production, notamment pour les œuvres de circonstance. En fait, la " servitude » du Rhétoriqueur ne s'étend pas forcément à son œuvre littéraire. D'après Thiry, au lieu de parler d'u aliénation» et de "servitude », il faut plutôt introduire la notion de service princier, entendu comme un engagement profond, qui fait du poète non seulement un porte-parole du prince mais aussi son conseiller et mentor. De cette conception du rôle du poète découle sa grandeur, laquelle réside dans la portée de 
la tâche qu'il doit accomplir. Si le rhétoriqueur explore la langue c'est aussi, ou peutêtre surtout, pour forger un discours à la mesure de ses ambitions et pour ennoblir un art qu'il voue à une élite intellectuelle. Nous voilà loin de l'image du poète rhétoriqueur aliéné :

\begin{abstract}
Le rhétoriqueur n'est pas seulement un simple «manœuvrier du verbe » ou un « jongleur de syllabes», uniquement soucieux d'ingénieux montages qui révèlent au critique d'aujourd'hui sa révolte - sans que lui-même ait toujours pris conscience de celle-ci, et sans que les princes, érudits et grands officiers à qui il destinait d'abord ses œuvres l'aient aperçue. C'est aussi un homme, avec son cœur, ses sentiments, ses idées, plus orienté certes vers le service de la Cité que vers celui des dames (Thiry, $1978: 100$ ).
\end{abstract}

En dépit de ces réserves, il faut reconnaître l'importance capitale des travaux de Zumthor pour l'histoire de la critique littéraire concernant les Grands Rhétoriqueurs. Ce dernier a tracé une voie nouvelle - certes parfois risquée - de l'analyse du texte «rhétoriqueur » en proposant tout un éventail de possibilités méthodologiques. Mais ce qui mérite notre attention avant tout, c'est qu'il a montré que cet ensemble de textes autrefois hermétiques et oubliés pouvait donner lieu à une interprétation cohérente. Son mérite consiste donc dans une rupture définitive avec une tradition tendancieuse qui n'a pas voulu reconnaître aux Rhétoriqueurs leur titre de noblesse. En suscitant une polémique, ses travaux ont fait progresser la recherche dans ce champ, autrefois sous-estimé, de la poésie française.

C'est surtout la critique thématique qui s'est avérée fructueuse. Les suggestions de Thiry se sont vérifiées dans la pratique de l'analyse des textes. Les poètes rhétoriqueurs, aussi bien ceux de cour que les participants des Puys ont un message à transmettre, message philosophique et esthétique. Limiter leur effort à un jeu verbal serait méconnaître la conception du rôle de l'écrivain dans la société à laquelle ils s'identifiaient.

Les années quatre-vingt-dix sont marquées par l'intensification des efforts pour élucider les questions épineuses de la grande rhétorique. Les chercheurs ont suivi deux axes principaux, l'un examinant les propositions déjà lancées par Zumthor et promouvant l'analyse des œuvres des Rhétoriqueurs dans leur ensemble, ou une redécouverte des poètes oubliés; l'autre s'intéressant davantage à une thématique pratiquement ignorée jusqu'alors : la poésie des Puys.

Quand François Cornilliat, en 1989, a présenté sa thèse sur le rôle de l'ornement chez les Grands Rhétoriqueurs, il était évident que la bataille décisive pour leur asssurer une place digne parmi les poètes français avait été gagnée. Ce livre imposant fait date dans la critique littéraire par sa richesse documentaire mais aussi par la finesse de ses analyses (Cornilliat, 1994).

Les publications foisonnent, la poésie des Grands Rhétoriqueurs cesse de provoquer un sourire méprisant ou des remarques ironiques. Elle commence à inspirer plusieurs chercheurs. Michel Zink, dans son introduction à Grands Rhétoriqueurs, publication du Centre V. L. Saulnier, insistait sur la spécificité de cette poésie :

Chez les Grands Rhétoriqueurs tout est paradoxe : leur dénomination inappropriée et inévitable; les frontières floves qui entourent plutôt mal que 
bien leur troupe hétéroclite ; leur art littéraire, aussi friand de l'inattendu que de la norme (Zink, 1997 : 7).

Cet ouvrage présente des communications variées dont certaines nous semblent ici particulièrement intéressantes à relever : ainsi, J. Cerquiglini a porté sa réflexion sur l'esthétique des Grands Rhétoriqueurs en soulignant l'importance de la notion de "langue esclatante » qui caractérise leur poétique; Pierre-Yves Badel a analysé la spécificité du rondeau au temps de Jean Marot; T. Mantovani s'est intéressé au problème de l'abolition de la coupe féminine traité par Gratien du Pont dans son Art et science de rhetoricque metriffiee; J. Devaux a consacré sa communication à la question des rapports entre la politique et la rhétorique en examinant le cas de Jean Molinet; C. Brown a analysé les affinités entre le texte et la miniature d'après l'exemple de l'œuvre de Pierre Gringore.

Les études consacrées à la poésie palinodique progressent parallèlement. C'est à Gérard Gros que revient le mérite d'avoir fait découvrir à un public plus vaste la poésie mariale présentée aux concours des Puys du Nord de la France. II a réussi à faire sortir de l'oubli des poèmes parfois trop sophistiqués qui émanaient pourtant d'un besoin naturel d'écrire et d'exprimer ainsi des réflexions sur la foi et la vie. Le premier volume, intitulé Le poète, la Vierge et le Prince du Puy, est consacré au contexte socio-historique des concours poétiques (Gros, 1992); le deuxième, Le poème du Puy marial, aux genres pratiqués : le serventois et le chant royal (Gros, 1996). L'auteur souligne le caractère complexe de l'activité des confréries qui organisaient les concours :

À côté du rituel pieux et de la convivialité festive, la foi dans la rhétorique et
ses vertus singularise ce milieu citadin lesté de clergie, proche à tous égards
de l'artisanat somptueux des Grands Rhétoriqueurs plus que de l'idéal de
cour ou du foisonnement dévot dont s'achalandent bientôt les librairies. Ici
l'on cherche à faire de la difficulté du sujet et de la complexité de la forme
un argument esthétique. On croit à l'admiration littéraire comme on adhère
au mystère marial. Non que parfois le savoir faire ne s'avilisse en faire savoir,
la conviction ostensible en ostentation exclusive, ou que le mieux, voire le
trop, ne soit adversaire du bien et du bon (Gros, 1992:18-19).

Sans oublier la tendance vertigineuse aux jeux de langue si caractéristique des Grands Rhétoriqueurs, dont ce chercheur présente plusieurs exemples dans son étude Le Poète marial et l'art graphique (Gros, 1993). Poème abécédaire, poème personnalisé par l'acrostiche, poème dessiné, poème-rébus défilent en déclinant leurs possibilités de réjouir le lecteur. Le monde spirituel des Rhétoriqueurs n'est pas privé de joie ni d'humour.

Les recherches de Denis Hüe s'inscrivent dans le même cadre: ce dernier a publié en 2002 un livre consacré à la poésie palinodique à Roven embrassant la période de 1486 à 1550. Cette étude imposante est accompagnée de Petite anthologie palinodique où figurent des poèmes présentés aux concours du Puy de Rouen, inédits jusqu'alors. L'auteur prépare le lecteur aux difficultés qu'il peut rencontrer pendant la lecture :

Cette poésie religieuse est aussi, paradoxalement, une poésie profane : ce sont toutes les activités de l'homme que l'on verra représenter ici, dans une volonté gourmande de les inventorier et de les découvrir, de les exalter et d'exalter, dans Marie, I'homme qui se dépasse. Poésie profane, le terme est 
juste, en ce que nous sommes en un temps où toute pensée, toute attitude se définit comme en fonction de la foi, de l'église, du temple, du fanum qui a suscité ce mot (Hüe, 2002b : 10).

Comme on le voit, on est loin de l'ambiance d'incompréhension d'autrefois. En guise de conclusion, citons un fragment de l'Introduction de Thierry Mantovani aux actes du colloque organisé par CÉRÉDI à l'Université de Roven, dont le thème était «Première poésie française de la Renaissance. Autour des Puys poétiques normands $»$ :

\begin{abstract}
Les pratiques de lecture ont changé et, fort heureusement, nous ne lisons plus les rhétoriqueurs ou les poètes palinodistes comme Henri Guy. Les trois dernières décennies, cependant, n'ont pas été statiques : à l'euphorie un peu révolutionnaire des années soixante-dix qui appliquait volontiers à l'étude des textes une grille d'analyse articulée sur la dialectique oppression/subversion (voyez P. Zumthor) a succédé une approche plus mesurée, plus sereine peut-être. Bien que se nourrissant de nos sensibilités d'hommes et de femmes de la fin du XXe siècle - pourrait-il en être autrement? -, elle aborde les œuvres en contexte et, autant qu'il est possible, se garde de la tentation plus ou moins consciente d'une annexion aux préoccupations contemporaines (Arnould \& Mantovani, $2003: 11$ ).
\end{abstract}

Les métamorphoses de l'attitude des critiques littéraires envers la poésie des Grands Rhétoriqueurs ont permis d'élargir le champ de recherche, stimulant de la sorte des investigations de plusieurs jeunes chercheurs. En effet, cette histoire prouve, une fois de plus, que la liberté dans la recherche scientifique reste fondamentale si on veut jouir d'un progrès réel dans le domaine de la critique littéraire.

\title{
Bibliographie
}

ARnould, J-C. \& MAntovanI, T. (ed.). (2003). Première poésie française de la Renaissance. Autour des Puys poétiques normands. Paris : Champion.

CERQUIGLINI, J. (1997). L'Éclat de la langue. Éléments d'une esthétique des Grands Rhétoriqueurs. In SAULNIER, C. V. L. Grands Rhétoriqueurs, 14. Paris: Centre V. L. Saulnier Université Paris-Sorbonne, Presses de l'École Normale Supérieure, pp. 75-82.

CORnilliat, F. (1994). "Or ne mens \#. Couleurs de l'éloge et du blâme chez les "Grands Rhétoriqueurs ». Paris : Champion.

CREPET, É. (1861). Les poètes français: recueil des chefs-d'œuvre de la poésie française depuis les origines jusqu'à nos jours, avec une notice littéraire sur chaque poète. T. I : du XIle au XVle siècle, Paris: [Maison Quantin].

GOYET, F. (1990). Traités de poétique et de rhétorique de la Renaissance. Paris : LGF.

Gros, G. (1992). Le poète, la Vierge et le prince du Puy. Étude sur les Puys marials de la France du Nord du XIVe siècle à la Renaissance. Paris : Klincksieck.

- (1993). Le poète marial et l'art graphique. Caen : Paradigme.

- (1996). Le poème du Puy marial, Paris : Klincksieck. 
GuY, H. (1910). Histoire de la poésie française au XVIe siècle. Tome I: L'école des rhétoriqueurs. Paris : Champion.

HüE, D. (2002a). La poésie palinodique à Roven (1486-1550), Bibliothèque littéraire de la Renaissance, série III, XLIV. Paris : Champion.

- (2002b). Petite anthologie palinodique (1486-1550). Paris : Champion.

JODOGNE, P. (1970). Les "rhétoriqueurs" et l'Humanisme. Problème d'histoire littéraire. In LEVI, A. H. T. Humanism in France at the end of the Middle Ages and in the early Renaissance, Manchester/New York, pp. 150-175.

Petit De JulleVille, L. (ed.). (1897). Histoire de la langue et de la littérature française des origines à 1900, Paris : Armand Collin.

Saulnier, C. V. L. Grands Rhétoriqueurs, 14, Paris : Centre V. L. Saulnier, Université de Paris-Sorbonne, Presses de l'École Normale Supérieure.

SCHMIDT, A.-M. (1958). "L'Age des Rhétoriqueurs », dans le chapitre "La Littérature humaniste à l'époque de la Pléiade ॥, in Histoire des littératures III, Gallimard, Bibliothèque de la Pléiade, pp. 175-190.

THIRY, C. (1978). Lecture du texte de «rhétoriqueur », Cahiers d'Analyse textuelle, 20, pp. 85-101.

- (1980). La poétique des Grands Rhétoriqueurs. A propos d'un ouvrage récent, Le Moyen Age, 86 (1), pp. 117-133.

ZUMTHOR, P. (1978a). Anthologie des Grands Rhétoriqueurs. Paris : Union Générale d'Éditions.

- (1978b). Le masque et la lumière. La poétique des grands rhétoriqueurs. Paris : Seuil. 